\title{
Study of laser bending of a preloaded Titanium alloy sheet
}

\author{
Xiufeng Wang ${ }^{1}$, , Dongxu Liu ${ }^{2}$, Weidong $\mathrm{Li}^{1}$, Lang $\mathrm{Xu}^{1}$, and Yaoguo Dong ${ }^{1}$ \\ 1 School of Mechanical Engineering and Automation, Beihang University, Beijing 100191, P.R. China \\ 2 CAE Division, Beijing Computing Center, Beijing 100094, P.R. China
}

Received 30 December 2014 / Accepted 23 January 2015

\begin{abstract}
Laser bending of sheet metals with preload offers some attractive characteristics/merits, comparing to laser free bending without prestressing on the metals. The study reported in this paper was focused on a Titanium alloy which finds widespread applications in aerospace manufacturing. FE simulation of laser bending with prestressing on the Titanium alloy sheet was conducted for the analysis of the bending process and experiment carried out to verify the model and the result. It was shown that the simulation result is close to that measured in the experiment. Based on the computed result, the load-displacement curve was analysed and transmission efficiency of the elastic energy defined to evaluate the bending effect. These enhanced understanding of the mechanism of laser bending with a preload. A method for the optimization on technological parameters was further proposed. Referring to the deformation targeted, the preload value was determined through the FE simulation. The result showed that, on the premise that the specimen surface can be prevented from damaging, transmission efficiency of the elastic energy could reach to the maximum value through adjusting technological parameters of the laser system and deformation accuracy of the specimen could also be improved through this approach. The work presented in this paper may find its application in the manufacture of Titanium alloy sheets with a more cost-effective and a more precise way.
\end{abstract}

Key words: Preload, Titanium alloy sheet, Laser bending, FE simulation, Transfer efficiency of elastic energy

\section{Introduction}

Laser bending with preload is a new forming technique in which firstly, a pre-deformation in the sheet metal is produced by a clamp, and then the region with elastic energy concentration in the part is scanned by a laser beam along a certain path. As a result of the laser thermal effect, deformation resistance of the material is reduced and the elastic energy transferred into plastic energy in the scanned area. As a consequence, the sheet metal could be deformed accurately. The deformation can be controlled through adjusting type and magnitude of the preload, laser technological parameters, depending on the elastic energy storage capability in the scanned area and its transformation efficiency. The process is an ideal option for forming Titanium alloy sheets, which are difficult to be formed at a room temperature due to poor plasticity, low elastic modulus and large springback at such a temperature. It is expected that mature development of such a forming technique could provide a novel method for the bending of Titanium sheets for practical applications and could contribute significantly to the development of new forming technologies.

Referring to the recently published research papers, the work mainly dealt with subjects such as influencing factors

*e-mail: wangxiufeng@buaa.edu.cn in bending processes, mechanisms of variations and changes in microstructure of Titanium alloy sheets in laser bending [1-6]. There was seldom work concerning laser bending with preload. The laser bending process with the auxiliary load was simulated on ship-plate material D36 and stainless steel, and their deformations were predicted [7, 8]. Laser bending with preload of the complex structure with an aluminum alloy plate was researched in which influencing factors and material properties changes during the bending process were analysed [9]. A feasibility experiment on a Ti-6Al-4V sheet was conducted and main influencing factors were analysed $[10,11]$.

At present, many parts used in aircrafts, satellites and rockets are formed Titanium alloy parts the shapes of which may be simple: straight line bending and curve bending. Traditional bending techniques would normally require high-temperature resistance dies, the size of parts may also be restricted by the furnace capacity [12], etc. While laser bending with preload offers many potential merits to the manufacture with Titanium alloy sheets, there is still need to further fundamental understanding on the process, material and part geometry to be formed, in order to achieve better process control for better quality and efficiency. For this consideration, FE simulation, combined with forming experiment, was conducted to study laser bending with preload of a Titanium sheet. Numerical 


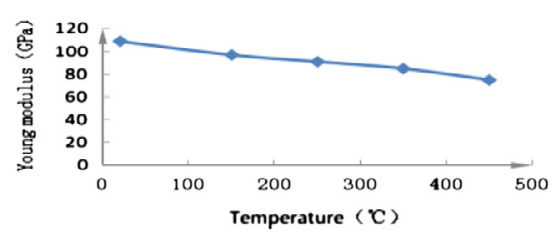

(a) Young modulus

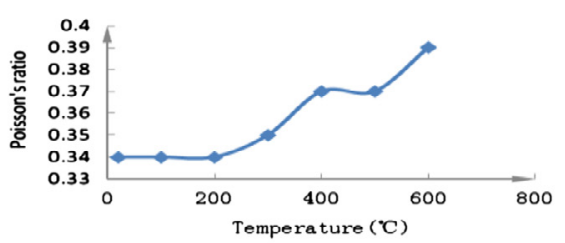

(d) Poisson' ratio

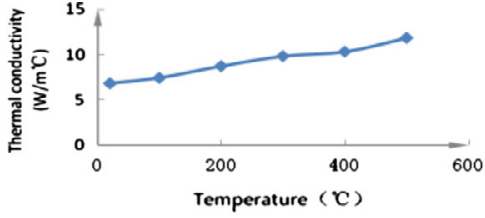

(b) Thermal conductivity

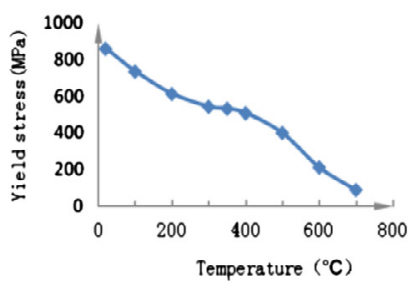

(e) Yield stress

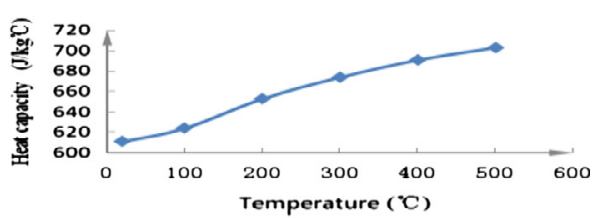

(c) Heat capacity

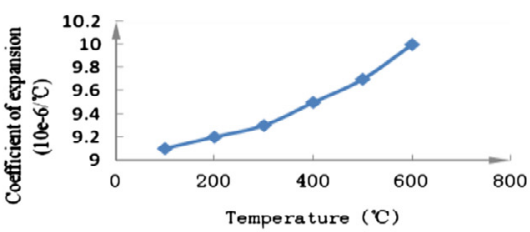

(f) Coefficient of expansion

Figure 1. Variation of thermal and mechanical properties of material versus temperature.

simulation of the laser prestressed bending was carried out using FE code ABAQUS, which was verified with the corresponding experimental result. The load-deformation curve was then obtained, the transformation efficiency of elastic energy defined, and the method of optimizing the technological parameters put forward.

\section{Process and FE simulation}

A Titanium alloy sheet Ti-6Al-4V was chosen the dimensions of which are $70 \mathrm{~mm} \times 30 \mathrm{~mm} \times 1 \mathrm{~mm}$. Firstly, the specimen was loaded laterally with a clamp to form an arch, and the lateral displacement being $4 \mathrm{~mm}$ and $9 \mathrm{~mm}$ respectively. Laser is then scanned on the surface of the specimen along the arch height where is the elastic energy concentration area. Laser power output used was $1000 \mathrm{~W}$, scanning speed $20 \mathrm{~mm} / \mathrm{s}$ and laser spot diameter $5.5 \mathrm{~mm}$. The specimen cooled down in air to obtain the bending angle.

The process was simulated with a Galerkin weighted residual Method to solve the instantaneous heat transfer equation (ABAQUS software). Temperature-depended thermo-physical properties of the material are shown in Figure 1 [13].

\subsection{FE modelling}

The specimen was discretized by means of eight-node linear brick elements with a view to attaining a high computational precision, considering that a high temperature gradient will be generated in the heated region, when laser beam irradiates on the specimen surface. The element size is $0.5 \mathrm{~mm} \times 0.5 \mathrm{~mm} \times 0.2 \mathrm{~mm}$, being shown in Figure 2 .

The analysis process was divided into two steps, namely, the preload clamping process and the laser scanning process.

(1) Preloading by clamping

The initial lateral displacement of the specimen was regarded as a control variable and the elastic energy distribution of the specimen then obtained. When the preload

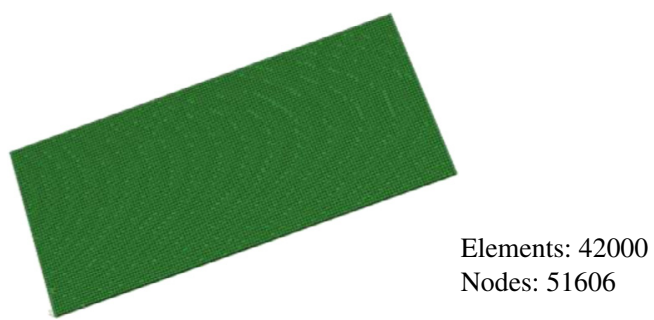

Figure 2. FEM model of calculation.

was large enough, a plastic energy was generated inside the specimen to a certain extent. Two conditions were studied: when the lateral displacement is $4 \mathrm{~mm}$, specimen is formed to an arch height of $5.5 \mathrm{~mm}$, and when the lateral displacement is $9 \mathrm{~mm}$, the specimen is formed to an arch height of $12 \mathrm{~mm}$.

(2) Laser scanning

The Gaussian distribution of the laser spot was adopted. Laser beam was treated as an input in a form of an additional heat flux vector and its action position changes with time simultaneously. Laser beam continuous irradiation was treated as jumping and intermittent moving at microstep intervals, and the action position of the heat flux vector only changes with time simply [14]. The calculated steps are shown as follows:

It is supposed that the width of the sheet metal $x$ is integral multiple of the mesh width $\Delta x$. Laser spot moves with a $\Delta x$ distance and remains a $t_{p}$ time during laser continuous irradiation, then

$$
t_{p}=\frac{\Delta x}{v}
$$

where $v$ is laser scanning speed.

The laser beam travels for a spot distance after $d / \Delta x$ jumps. When the value of $\Delta x$ is extremely small, the laser beam continuous moving can be assumed and then simulated. The heat flux density distribution in a spot is regarded to be uniform within each $t_{p}$, then 

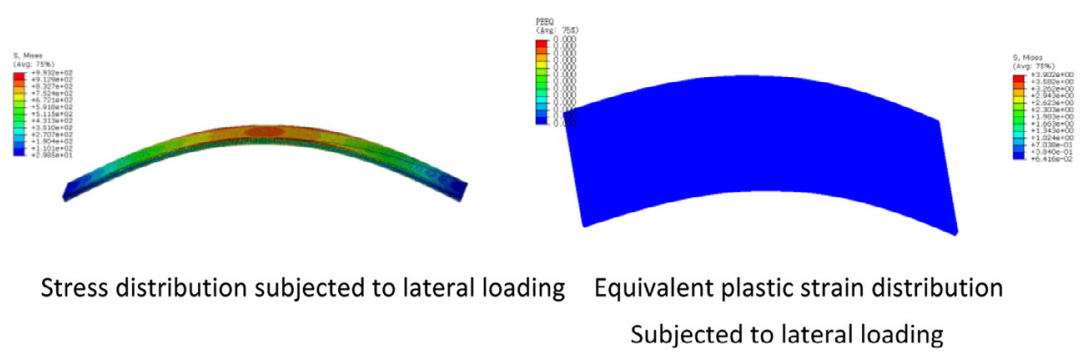

Stress distribution after unloading

(a) Specimen with initial lateral displacement of $4 \mathrm{~mm}$

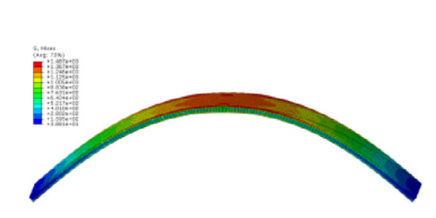

Stress distribution subjected to lateral loading

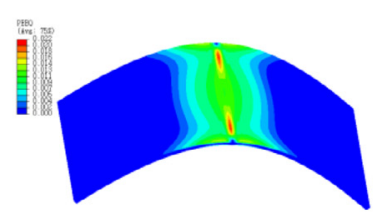

Equivalent plastic strain distribution Subjected to lateral loading

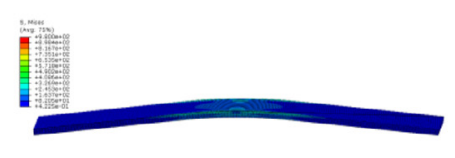

Stress distribution after unloading

(b) Specimen with initial lateral displacement of $9 \mathrm{~mm}$

Figure 3. Simulation of lateral loading and unloading without laser scanning.

$$
q=\frac{4 A P}{\pi d^{2}}
$$

where $A$ is the absorption coefficient of sheet metal $(A=0.2)$, and $P$ is laser output power.

The process of laser beam moving crossing the specimen surface is simulated by considering automatic loading by means of DFlux subroutine in ABAQUS software.

The specimen cools in the air after laser irradiation. $\beta$ is regarded as a total heat exchange coefficient including convection and radiation. The lost energy due to heat transfer can be calculated with the following formula:

$$
q_{h}=\beta\left(T-T_{0}\right)
$$

where $T_{0}$ is initial temperature $\left(T_{0}=20{ }^{\circ} \mathrm{C}\right.$ as a room temperature).

The subroutine dealing with total heat-exchange boundary was programmed with FORTRAN language and $\beta$ value versus temperature was defined by a FILM subroutine.

\subsection{Simulation results}

(1) A simulation result of lateral loading and unloading is shown in Figure 3.

Figure 3 shows the stress and equivalent plastic strain distribution of the specimen under lateral loading and after unloading. The maximum stress and equivalent plastic strain mainly occur at the top section of the arch. In Figure 3a, the maximum stress is $993.2 \mathrm{MPa}$ and the arch height $5.5 \mathrm{~mm}$. The equivalent plastic strain distribution PEEQ $=0$ indicates that only elastic deformation is produced inside the specimen. After preload is removed, specimen returns back to its original planar state, i.e., the specimen is loaded under a completely elastic state.
In Figure 3b, the maximum stress is $1487 \mathrm{MPa}$ and the arch height $12 \mathrm{~mm}$. The equivalent plastic strain distribution $\mathrm{PEEQ}=0.022$ indicates that partly plastic deformation occurs in the specimen. After preload is removed, the lateral displacement of the specimen decreases to $6.14 \mathrm{~mm}$ and arch height to $3.22 \mathrm{~mm}$, thus, the plastic deformation exists in the specimen.

(2) A simulation result of laser scanning is shown in Figure 4.

Figure 4 shows temperature versus time, stress distribution in the specimen under the laser scanning along the width direction and after unloading. It is obvious that the maximum stress decreases because the yield limit of the material drops at the elevated temperature, thus, more plastic deformation occurs with a similar level of initial lateral loading. In Figure $4 \mathrm{a}$, the specimen is in an elastic state, its maximum stress drops to $394.9 \mathrm{MPa}$. The bending angle of the specimen is from $140.5^{\circ}$ to $145^{\circ}$ due to the springback of the specimen. However, in Figure $4 \mathrm{~b}$, the specimen is in a partial elastic state, its maximum stress drops to $489.5 \mathrm{MPa}$, and bending angle of the specimen being from $120.3^{\circ}$ to $121.3^{\circ}$. From the comparison, the extent of bending obtained by the latter is larger than the former. The latter is applied with more preload which produces more elastic deformation that is later transformed into more plastic deformation.

\section{Bending experiment}

The experiment was carried out in the Institute of Laser Engineering, Beijing Polytechnic University. The scheme of the experiment is shown in Figure 5. 


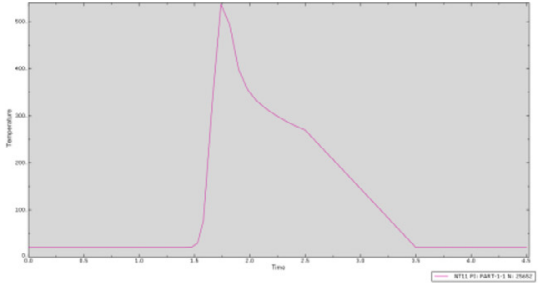

Temperature versus time

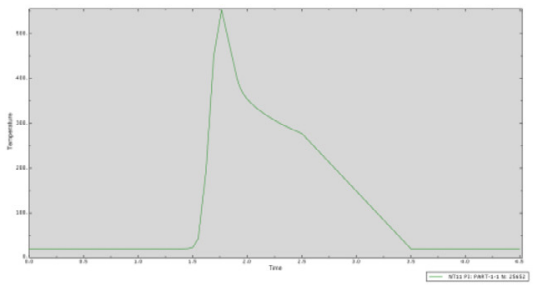

Temperature versus time

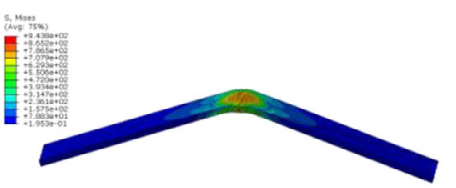

Stress distribution after Laser scanning

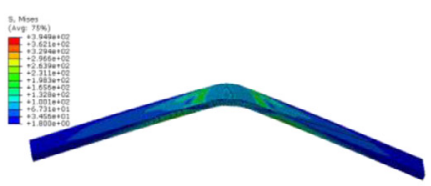

Stress distribution after unloading

(a) Specimen with initial lateral displacement of $4 \mathrm{~mm}$

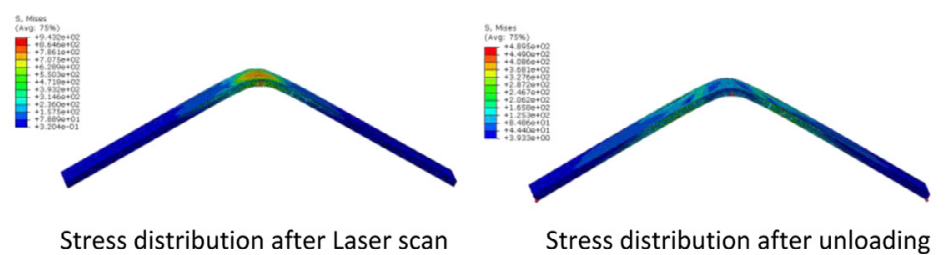

(b) Specimen with initial lateral displacement of $9 \mathrm{~mm}$

Figure 4. Simulation of laser bending with preload.

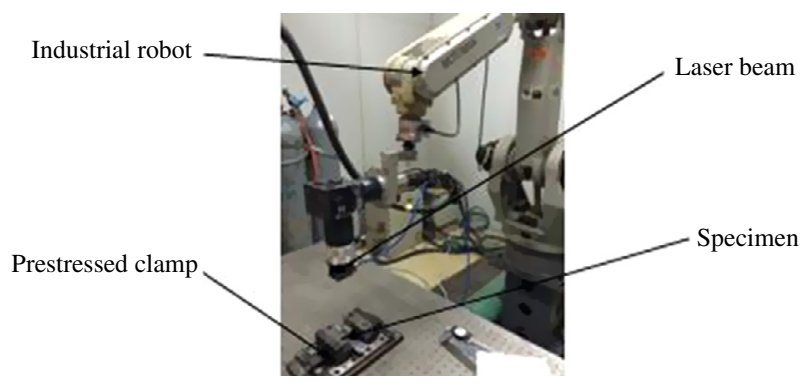

Figure 5. Scheme of the experiment.

The setup (Figure 5) includes a Nd-YAG $2000 \mathrm{~W}$ laser beam, an industrial robot, a preload clamp and a specimen. The specimen material and size are the same as that used in the simulation. Firstly, the specimen was loaded laterally with the preload clamp to form the arch shape. Then, the laser scanned over the surface of the specimen along the arch height where the elastic energy concentration area existed. Experimental parameters were set to be same as that computed. The specimen cooled down in air to obtain the final bending angle, as shown in Figures 6 and 7.

\section{Comparison of the experimental and simulation results}

A comparison of the two simulated results with experimental ones are shown in Figure 8.

Figure 8 indicates that the simulated results are approximate to the experimental ones. Therefore, the FE model can be regarded as a correct one with reasonable laser technological parameters had been chosen.

A load-deformation curve during laser bending with preload is shown in Figure 9.
Figure 9 shows that at point A, the specimen is preloaded with a force of $F_{\mathrm{A}}=29,796 \mathrm{~N}$ when the specimen is pushed to move laterally for $4 \mathrm{~mm}$ which leads to the specimen to be in a state of complete elasticity. After the laser scanning, the load drops to the point $\mathrm{C}, F_{\mathrm{C}}=11,847 \mathrm{~N}$. After releasing the clamp, the plastic deformation of the specimen along the horizontal direction is $X_{\mathrm{OE}}=2.4 \mathrm{~mm}$. Point B is preloaded with a force of $F_{\mathrm{B}}=44,610 \mathrm{~N}$ when the specimen is forced to move laterally for $9 \mathrm{~mm}$ which leads to the specimen to be in a state of partial plasticity. After the laser scanning, the load drops to the point $\mathrm{D}, F_{\mathrm{D}}=14,685 \mathrm{~N}$. After releasing the clamp, the plastic deformation of the specimen along the horizontal direction is $X_{\mathrm{OH}}=7 \mathrm{~mm}$.

The transfer efficiency of elastic energy can be defined as $\eta$ :

$$
\begin{gathered}
\eta_{\mathrm{A}}=\frac{S_{\triangle \mathrm{AOEC}}}{S_{\triangle \mathrm{AOG}}} \times 100 \% \\
=\frac{29,796 \times 4-11,847 \times 1.6}{29,796 \times 4} \times 100 \%=84 \% \\
\eta_{\mathrm{B}}=\frac{S_{\triangle \mathrm{BFHD}}}{S_{\triangle \mathrm{BFI}}} \times 100 \%=\frac{44,610 \times 6-14,685 \times 2}{44,610 \times 6} \times 100 \% \\
=89 \%
\end{gathered}
$$

Thus, the greater is the preload, the higher is the transfer efficiency of elastic energy $\eta$, and the bending effect is more significant as well. Therefore, the preload is expected to increase as much as possible in order to obtain the maximum bending effect, on the premise that the specimen surface quality is ensured.

The simulation results show that: (1). decreasing position of the point $\mathrm{C}$ is an effective way to increase $\eta$. That is to say that, when the specimen surface is maintained in a good condition, to increase laser power output or to decrease laser scanning speed as much as possible could lead to an increase of the maximum temperature in the spacemen and hence, further more elastic 


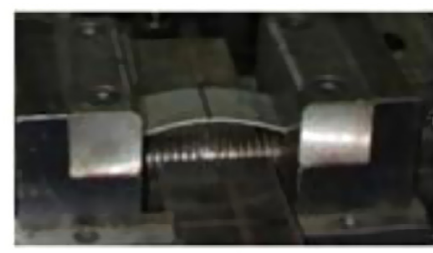

(a) Lateral loading

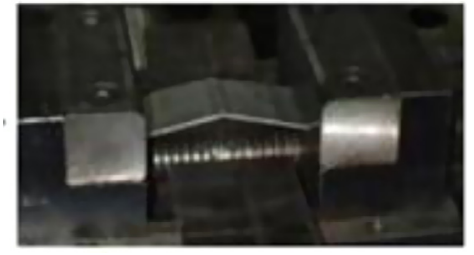

(b) Laser scanning at the arch height

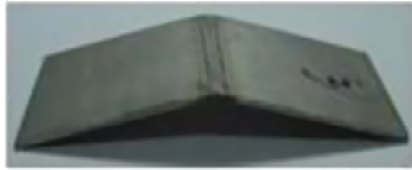

(c) Bending angle $149^{\circ}$

Figure 6. Laser bending with initial, specimen lateral displacement of $4 \mathrm{~mm}$.

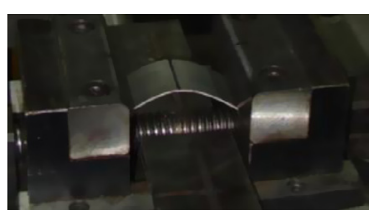

(a) Lateral loading

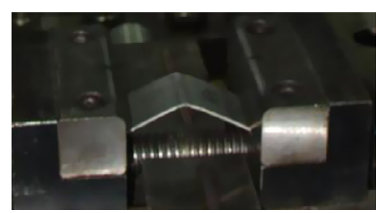

(b) Laser scan at the arch height

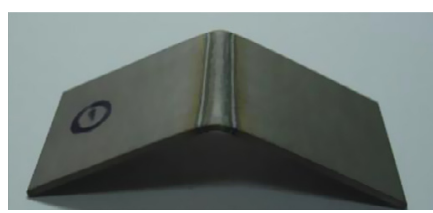

(c) Bending angle $121^{\circ}$

Figure 7. Laser bending with initial, specimen lateral displacement of $9 \mathrm{~mm}$.

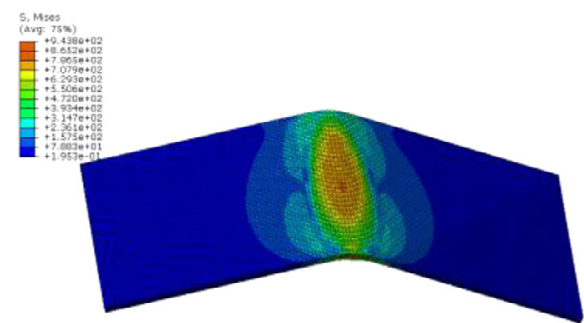

Simulated bending angle $145^{\circ}$

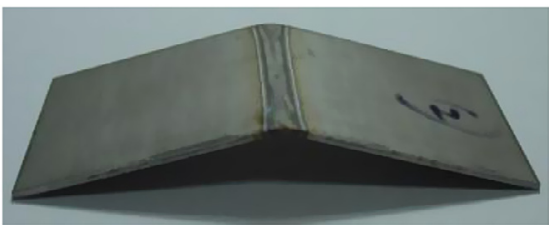

Experimental bending angle $149^{\circ}$

(a) Initial lateral displacement $4 \mathrm{~mm}$

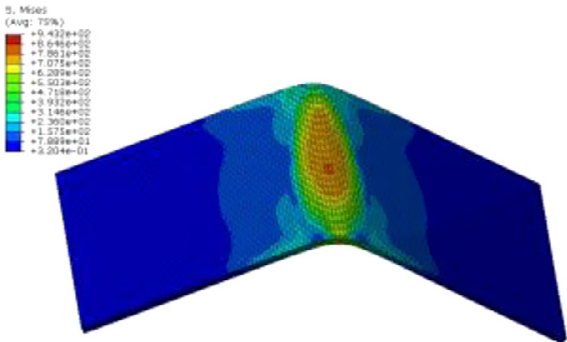

Simulated bending angle $121.3^{\circ}$

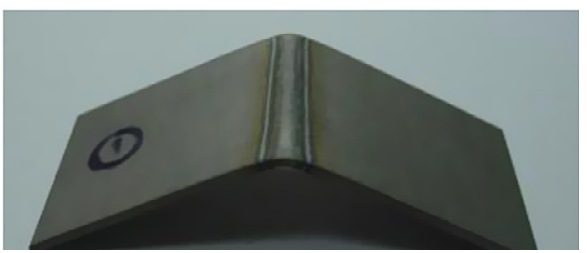

Experimental bending angle $121^{\circ}$

(b) Initial lateral displacement $9 \mathrm{~mm}$

Figure 8. Comparison of the simulated results with experimental ones.

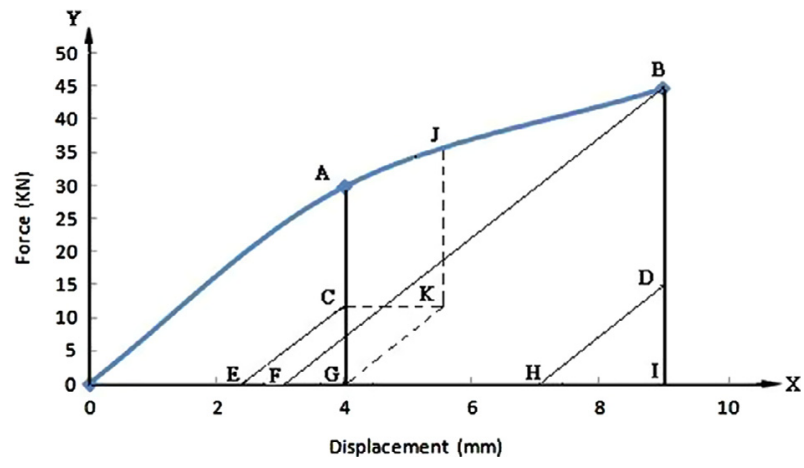

Figure 9. Load-deformation curve during laser bending with preload. deformation will be transferred into plastic deformation; and (2) when the preload is increased to a certain extent, the bending angle accuracy can be improved significantly. For example, if the preload $F_{\mathrm{A}}$ is increased to $F_{\mathrm{J}}$, as shown in Figure 9, the load will drop to the point $\mathrm{K}$ after the laser scanning, which is the same line as the point $\mathrm{C}$. After unloading, specimen obtains the required amount of the deformation $X_{\mathrm{OG}}$.

\section{Conclusions}

From the study reported above the following conclusions may be drawn:

(1) Simulation of laser bending with preload on a Titanium sheet is accurate enough for conducting investigation into this process with this modelling tool. 
(2) Stresses and deformations in the laser bending with preload have been obtained through the simulation from which the forming mechanism is better understood.

(3) The laser thermal effect enables to reduce the resistance of deformation during bending and can help to transfer elastic deformation energy into plastic deformation energy. The transfer effect can be measured with transfer efficiency of elastic energy. It is essentially different from laser bending alone.

(4) Laser thermal effect is significantly different under different preloads. Larger preload is more helpful in terms of getting more plastic deformation and higher bending accuracy, if surface quality of the specimen can be ensured.

(5) The transfer efficiency of elastic energy can be optimised through adjusting laser technological parameters.

Acknowledgements. The authors would like to acknowledge X.B. Wang, J. Guo and Yan Liang in the Institute of Laser Engineering, Beijing Polytechnic University, China, for their assistance during the experimental stage of this work.

\section{References}

1. L.Q. Li, Y.B. Chen, L.W. Zhang, X.S. Feng, Laser bending characteristics of Ti-6Al-4V Titanium alloy, The Chinese Journal of Nonferrous Metals 15 (2005) 842-848 (in Chinese).

2. P. Zhang, B. Guoa, D.-B. Shan, Z. Ji, FE simulation of laser curve bending of sheet metals, Journal of Materials Processing Technology 184 (2007) 157-162.
3. J. Yu, Study on numerical simulation and optimization design of laser bending of Titanium alloy sheets, Master Thesis, Hefei Polytechnic University, 2009 (in Chinese).

4. Y. Xiang, L. Hu, D. Peng, X. Huijuan, Research on Manufacturing Technology 5 (2010) 20-25 (in Chinese).

5. C. Liu, S.H. Liu, J.L. Liu, K.P. Hu, P. Xiao, Study on shape and size precision control of Titanium alloy in laser bending, Applied Laser 31 (2011) 50-56.

6. D.P. Shidid, M. HoseinpourGollo, M. Brandt, M. Mahdavian, Study of effect of process parameters on Titanium sheet metal bending using Nd: YAG laser, Optics \& Laser Technology 47 (2013) 242-247.

7. Z.Q. Yao, H. Shen, Y.J. Shi, J. Hu, Numerical study on laser forming of metal plates with pre-loads, Computational Materials Science 40 (2007) 27-32.

8. J. Liu, S. Sun, Y. Guan, Numerical investigation on the laser bending of stainless steel foil with pre-stresses, Journal of Materials Processing Technology 209 (2009) 1580-1587.

9. G. Chen, Chinese Journal of Lasers 38 (2011) 0601006-10601006-11 (in Chinese).

10. Y.C. Zhang, X.F. Wang, X.B. Wang, J. Guo, Laser prestressed bending of Titanium alloy sheet, Applied Mechanics and Materials 633-634 (2014) 773-776.

11. Z.C. Shen, Experimental research on laser forming for V shape part, Bachelor thesis, Beihang University, 2014.

12. P.L. Li, Z. Zhang, Thermoforming mold for Titanium alloy, Aeronautical Manufacturing Technology 21 (2012) 94-97 (in Chinese).

13. China Standard Press. Chinese Aviation Materials Handbook (Second Edition), vol. 4, 2002, pp. 104-132 (in Chinese).

14. Z. Ji. Laser sheet metal forming and Simulation, Ph.D dissertation, Northwestern Polytechnic University, Xi'an, 1997. 